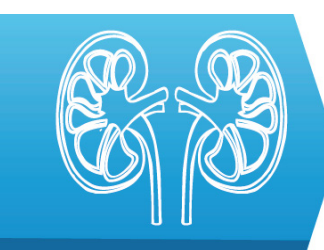

UROLOGY

\title{
Laparoscopic radical cystectomy and nephroureterectomy en bloc resection with lomboaortic and pelvic lymph node dissection
}

\author{
Bogdan Petruț ${ }^{1,2,3}$, Roxana-Andra Coman², Vlad Hârdo ${ }^{3}$, Bogdan Coste ${ }^{3}$, \\ Teodor Maghiar ${ }^{3}$
}

1) Urology Department, Iuliu Hațieganu University of Medicine and Pharmacy, Cluj-Napoca, Romania

2) Urology Department, "Prof. Dr. Ion Chiricuţă" Oncology Institute, ClujNapoca, Romania

3) Urology Department, Pelican Hospital, Oradea, Romania
DOI: $10.15386 / \mathrm{mpr}-1626$

Manuscript received: 11.03.2020

Received in revised form: 11.05 .2020

Accepted: 01.06.2020

Address for correspondence:

dr.roxanacoman@yahoo.com

This work is licensed under a Creative Commons Attribution-NonCommercialNoDerivatives 4.0 International License

\begin{abstract}
Background and aims. In patients with recurrent high grade or muscle-invasive bladder cancer and concomitant upper urinary tract tumors or non-functional kidney laparoscopic radical cystectomy and nephroureterectomy with lomboaortic and pelvic lymph node dissection can be performed. We present our initial experience.

Methods. Between 2018 and 2019, 4 patients underwent laparoscopic radical cystectomy and unilateral nephroureterectomy en bloc resection with lomboaortic and pelvic lymph node dissection. The nephroureterectomy was the first part of the surgery. It was followed by radical cystectomy with lymphadenectomy. All the specimens were removed en bloc in an endobag through a midline incision.

Results. The patients' demographic characteristics and perioperative outcomes were retrospectively collected and evaluated. All surgeries were completed laparoscopically. There was no need for conversion to open surgery. The mean operative time was $286,25 \mathrm{~min}$ with minimal blood loss $(260 \mathrm{ml})$. No major complications were reported. The mean follow-up period was 8.75 months.

Conclusion. Laparoscopic radical cystectomy and nephroureterectomy en bloc resection with lomboaortic and pelvic lymph node dissection can be safe and feasible in selected cases as an alternative approach to the open surgery, offering good oncological and functional results.
\end{abstract}

Keywords: laparoscopy, en bloc resection, radical cystectomy, radical nephroureterectomy, pelvic lymph node dissection

\section{Background and aims}

Urothelial carcinomas hold the fourth place among the most common cancers in developed countries. They are located more frequently in the urinary bladder and only $5-10 \%$ are located in the upper urinary tract [1]. Regarding upper urinary tract carcinomas, $17 \%$ of cases are also associated with bladder cancer [2]. In $2-6 \%$ the contralateral upper urinary tract is also affected [3].

Open radical cystectomy is still considered the standard treatment for muscle-invasive bladder cancer, high risk and recurrent non-muscle-invasive bladder tumors, BCG refractory, relapsing, and unresponsive T1G3 tumors
[4,5]. Open radical nephroureterectomy with bladder cuff excision is the standard treatment for high risk upper urinary tract carcinomas [6].

Simultaneous nephroureterectomy and cystectomy can be performed in patients with recurrent high grade or muscle-invasive bladder cancer and concomitant upper urinary tract tumors or non-functional kidney [7].

Since the first laparoscopic nephroureterectomy in 1991 by Clayman et al. [8] and since the first laparoscopic radical cystectomy in 1992 by Parra et al. [9] and also due to technical progress, laparoscopic radical nephroureterectomy or radical cystectomy have been reported 
to have a lot of advantages over open techniques in a high number of cases. Laparoscopic techniques offer fewer intraoperative and postoperative complications, decrease intraoperative blood loss, less need of analgesics, shorter hospital stay, and earlier recovery [10-12]. Also, minimally invasive approaches have demonstrated having comparable functional and oncological results with open surgery $[13,14]$. Several years after, Berglund et al. reported the feasibility of performing laparoscopic radical cystoprostatectomy and bilateral nephroureterectomy [15].

We present the outcomes and complications of our first 4 patients who underwent simultaneous laparoscopic radical cystectomy and nephroureterectomy with lomboaortic and pelvic lymph node dissection.

\section{Methods}

We present our first 4 patients who underwent radical cystectomy and nephroureterectomy en bloc resection with pelvic lymph and lomboaortic node dissection performed by laparoscopic approach between 2018 and 2019. The patients were operated by the same surgeon. Committee.

This study was approved by the local Ethical

All patients had previous histories of muscleinvasive or recurrent non-muscle invasive bladder cancer. The indications for individual surgeries were: recurrent high-grade bladder tumors with coexisting high grade papillary urothelial carcinoma in the renal pelvis in a surgically solitary kidney (case 1), muscle-invasive bladder cancer with hydroureteronephrosis nonfunctioning kidney due to distal ureteral obstruction (case 2), muscle-invasive bladder cancer with upper urinary tract carcinoma (case 3), and muscle-invasive bladder cancer with urothelial carcinoma located in the renal pelvis and in the distal ureter and invasive bladder cancer (case 4). The preoperative imaging information was that all the tumors were specimenconfined. We used the Clavien-Dindo classification to evaluate postoperative complications.

The patients' demographic characteristics, surgical technique, and perioperative outcomes were retrospectively collected and evaluated.

\section{Surgical technique}

All surgeries were performed by laparoscopic transperitoneal approach.

After general anesthesia, a catheter was placed into the bladder. The patient was placed in a lateral decubitus position. The operative region (the flank and the abdomen) was prepared and draped.

A $10 \mathrm{~mm}$ trocar was used as the camera port and it was placed lateral of the rectus sheath in the right lower quadrant. It was inserted through a $2 \mathrm{~cm}$ incision using the Hasson open technique. The other two $10 \mathrm{~mm}$ trocars were placed in line, in the pararectal area. For performing the right nephroureterectomy, we used an additional $5 \mathrm{~mm}$ trocar in the epigastric region for elevating the liver (Figure 1).
The colon was mobilized medially. The lower pole of the kidney was identified and dissected. The dissection was continued medially, the gonadal vessels were dissected, secured with Hem-o-lock clips and sectioned between. The ureter was isolated and the dissection was continued towards the renal pelvis. The renal artery and the renal vein were identified, clamped with Hem-o-lock clips, and sectioned between. A perifascial dissection of the kidney was performed with the preservation of the adrenal gland if there was not clinical and intraoperative suspicion of invasion. Then the kidney, including Gerota fascia, was completely freed and the ureter dissection was continued towards the ureterovesical junction.

By performing the surgery in a transperitoneal approach, a large working space allowed changing the camera position and the working trocars according to the area of interest. The caudal trocar was used for the camera position when the ligation of the renal pedicle was performed and the upper pole of the kidney was dissected. The cranial trocar was used for the camera position when the pelvic area was the field of interest. Retroperitoneal lymphadenectomy, including paracaval, retrocaval, and paraaortic lymph nodes was performed. The removed lymph nodes were placed in an endobag and were sent to pathology.

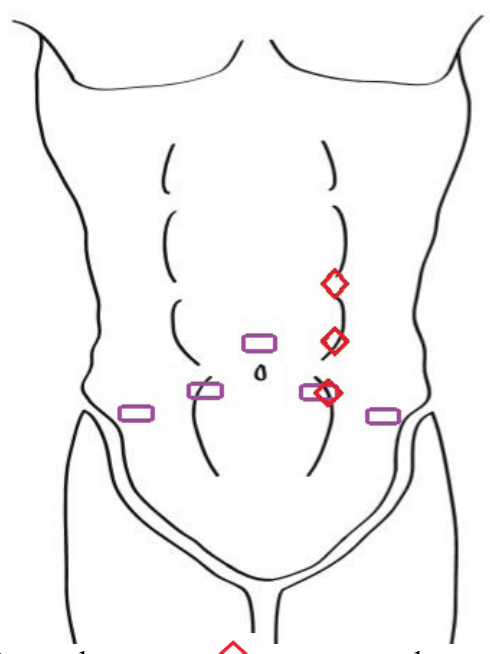

Figure 1. Ports placement: $\diamond$ represents the trocar placement for the laparoscopic nephroureterectomy and $\square$ represents the trocar placement for the laparoscopic cystectomy.

For the next step of the surgery, radical cystectomy and bilateral pelvic lymph node dissection, the patient was positioned in dorsal decubitus, in a Trendelenburg position.

The trocars used for radical cystectomy were placed under visualization. The camera trocar was placed supraumbilically. Two other $10 \mathrm{~mm}$ trocars were placed inline, inferior to the umbilicus, in the right and the left pararectal area, in a triangle fashion and two other $10 \mathrm{~mm}$ working trocars were placed in the left and 
right lower quadrant, proximal to the anterior-superior iliac spine (Figure 1). The camera trocar used for the nephroureterectomy would be used for the cystectomy as a working trocar.

\section{Male patients}

The obliterated umbilical artery and superior vesical pedicle were secured with Hem-o-lock clips and divided and the lateral plane was dissected, bilaterally. The posterior dissection was performed beneath the Denonvilliers fascia to the seminal vesicles up to the prostatic apex. The posterior and the lateral pedicles of the bladder were identified and divided.

An incision was performed in the peritoneum to mobilize the bladder from the anterior abdominal wall. The urachus and medial umbilical ligaments were incised and the space of Retzius was developed. The endopelvic fascia was divided bilaterally. The superficial dorsal venous complex was coagulated and divided. The puboprostatic ligaments were divided. The dorsal venous complex was secured using 3.0 V-Lock suture and the urethra was divided distal from the prostatic apex using cold scissors. A bladder catheter was inserted in the field and the balloon was inflated, used for drainage. The pelvic lymphadenectomy was performed around the iliac vessels and obturatory fosa bilaterally.

\section{Female patients}

The obliterated umbilical, superior vesical artery, the uterine and ovarian vessels were ligated using Hem-o-lock clips and divided. The round ligaments were transected, the uterus, fallopian tubes, and ovaries were dissected, but the uterus was kept attached to the vaginal vault to prevent loss of the pneumoperitoneum. Then, the posterior bladder wall dissection was performed and continued distally to the level of the bladder neck.

In order to mobilize the bladder from the anterior abdominal wall, an incision in the peritoneum was performed. The urachus and medial umbilical ligaments were incised and the space of Retzius was developed to the endopelvic fascia. The urethra was dissected circumferentially and transected with the anterior vaginal wall. The vaginal wall was closed transversally with an absorbable continuous suture. Then, the bilateral pelvic lymphadenectomy was performed.

The retroperitoneal approach was used to bring the transected ureter to the lateral abdominal wall. The ureter was implanted in the location of a laparoscopic port. The specimen (Figure 2 and Figure 3) was placed in an endobag, it was removed through a mid-line incision and it was sent to the pathology. The operative field was inspected for bleeding or injury.

The ureter was catheterized with a mono J stent which was secured with several sutures and the cutaneous ureterostomy was created.

The fascial layers were closed. The skin incisions were closed by intradermic suture.

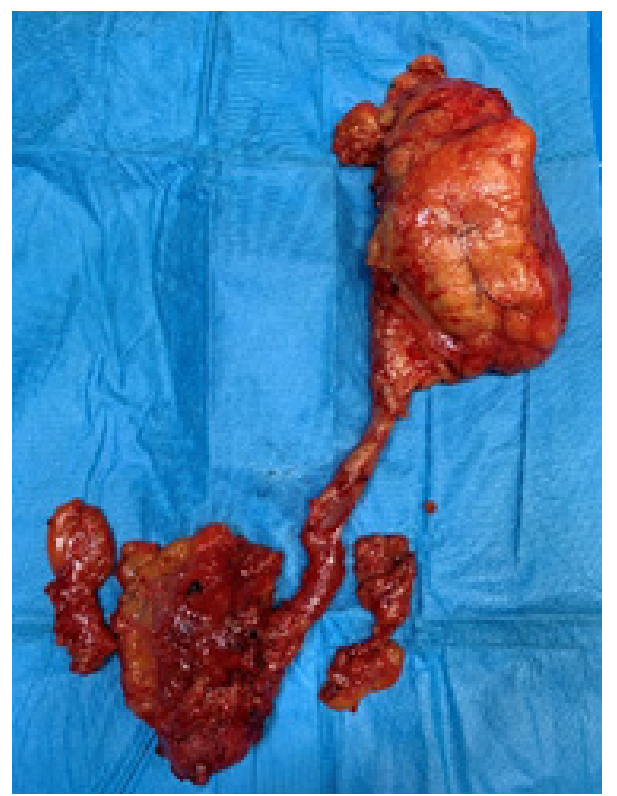

Figure 2. The specimen removed after laparoscopic radical cystoprostatectomy and left nephroureterectomy with pelvic lymph node dissection.

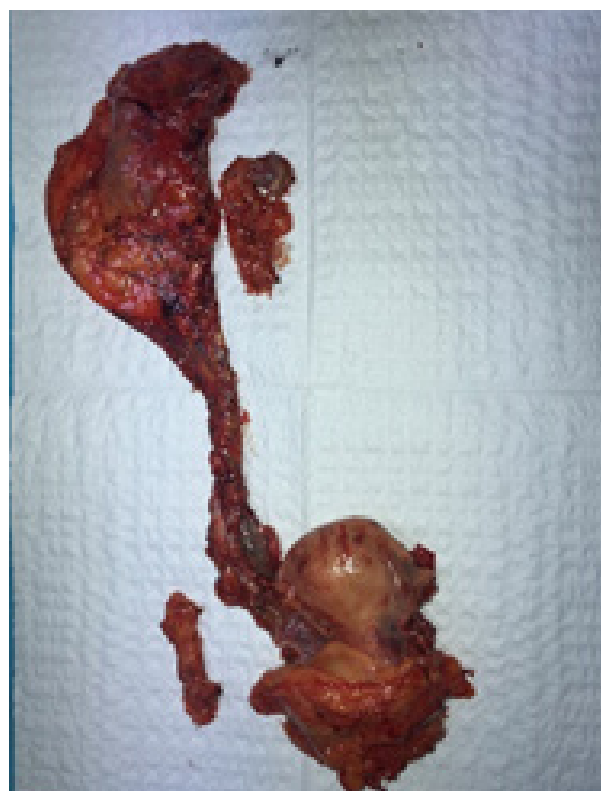

Figure 3. The specimen removed after laparoscopic radical cystectomy, hysterectomy, bilateral adnexectomy with right nephroureterectomy with lomboaortic and pelvic lymphadenectomy.

\section{Results}

The demographic patients' data and pathological results are presented in Table I. There were 2 males and 2 females who underwent laparoscopic radical nephroureterectomy and radical cystectomy. The patients' mean age was 66 years (range 59-73 years). 
Table I. Demographic patients' data and pathological results.

\begin{tabular}{|c|c|c|c|c|}
\hline & Case 1 & Case 2 & Case 3 & Case 4 \\
\hline Gender & F & $\mathrm{M}$ & F & M \\
\hline Age & 68 & 73 & 64 & 59 \\
\hline Year & 2018 & 2019 & 2019 & 2019 \\
\hline $\begin{array}{l}\text { Previous intravesical } \\
\text { BCG instillations }\end{array}$ & yes & yes & yes & no \\
\hline $\begin{array}{l}\text { Bladder pathological } \\
\text { stage }\end{array}$ & pT1G1 & pT2G3 & pT2G3 & pT2G3 \\
\hline $\begin{array}{l}\text { Upper urinary tract } \\
\text { location }\end{array}$ & $\begin{array}{l}\text { Right renal pelvis } \\
\text { Iliac and distal ureter }\end{array}$ & Left distal ureter & $\begin{array}{l}\text { Left renal pelvis } \\
\text { Left ureter }\end{array}$ & $\begin{array}{l}\text { Right renal pelvis } \\
\text { Distal ureter }\end{array}$ \\
\hline
\end{tabular}

Table II. Perioperative and postoperative data.

\begin{tabular}{l|cccc} 
& Case 1 & Case 2 & Case 3 & Case 4 \\
\hline No. of trocars & 8 & 7 & 7 & 8 \\
Side of surgery & Right & Left & Left & Right \\
Type of surgery & Laparoscopic radical nephroureterectomy and radical cystectomy & 270 & 285 \\
Perioperative time (min) & 280 & 310 & 210 & 230 \\
Blood loss (ml) & 350 & 250 & 6 & 5 \\
Hospital stay (days) & 8 & 5 & 4 & 4
\end{tabular}

Table III. Postoperative pathological stage.

\begin{tabular}{|c|c|c|c|c|}
\hline & Case 1 & Case 2 & Case 3 & Case 4 \\
\hline Bladder pathological stage & pT1G1 & pT3a G3 & pT2aG3 & pT2G3 \\
\hline $\begin{array}{l}\text { Upper urinary tract pathological } \\
\text { stage }\end{array}$ & pT3G3 & $\begin{array}{l}\text { Atrophic parenchima, } \\
\text { pyelonephritis }\end{array}$ & pT4G3 & pT1G2 \\
\hline $\begin{array}{l}\text { No of lomboaortic lymph nodes } \\
\text { dissected }\end{array}$ & 14 & 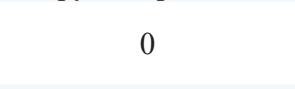 & 6 positive / 11 & 6 \\
\hline No of pelvis lymph nodes dissected & 13 & 17 & 16 & 14 \\
\hline
\end{tabular}

One patient (case 1) had a previous open radical nephroureterectomy and one patient (case 2) presented a hydroureteronephrotic nonfunctioning kidney, due to the recurrence of invasive bladder cancer of the left lateral bladder wall that involved the left ureteral orifice. The first three patients had received intravesical instillations of BCG immunotherapy and were followed up by regular cystoscopies. The upper urinary tract tumors sites were: renal pelvis in 3 cases, iliac ureter in 1 case and in 3 cases in the distal ureter. Left-side nephroureterectomy was performed in 2 patients and right-side nephroureterectomy was performed in 2 patients.

Perioperative and postoperative information are shown in Table II. There was no conversion to open surgery. The total mean operative time was 286,25 min (range 270-310 min) with a mean blood loss of $260 \mathrm{ml}$ (range 210-350 ml). One case required blood transfusion. The hospital stay was 6 days (range 5-8 days). Intraoperative and postoperative complications were no more than Clavien-Dindo classification grade II. The mean followup period was 8.75 months (range 4-20 months).

The postoperative pathological stages are represented in Table III. Regarding surgical margins, there were no positive surgical margins, the tumors were removed completely.

In case 3, the pathological result was pT4N2MxL1V1, urothelial tumor located into the left renal pelvis, left ureter and left bladder lateral wall which invades the left adrenal gland and has positive lymph nodes in the renal hilum and lombo-aortics. The patient is having adjuvant chemotherapy.

Case 4 was also described in the pathological result: prostatic adenocarcinoma Gleason $6(3+3)$, ISUP 1, pT2N0V0Pn0R0. 
The patient presented as case 1 was followed up by periodical imaging evaluation. The last imaging evaluation, performed in September 2019, revealed no signs of tumor recurrence and no lymph nodes.

In October 2019, the patient (case 1) was admitted in our service for surgical treatment of vaginal prolapse (rectocele) caused by modified pelvic diaphragm resistance due to cystectomy. We repaired the rectocele by transvaginal approach. The patient had a favorable postoperative evolution.

\section{Discussion}

We successfully performed laparoscopic radical nephroureterectomy and cystectomy en bloc resection with lomboaortic and pelvic lymph node dissection in all 4 cases. Minimally invasive surgical techniques have been recommended to minimize the complications and to improve the recovery [16].

Performing laparoscopically, in a single session nephroureterectomy and radical cystectomy with lymphadenectomy is a challenging surgery. In order to have oncological safety, intraoperatively, special care must be taken to avoid tumor spillage and the specimen should be extracted en bloc with the bladder neck closed in order to avoid contact of urine with the abdominal cavity and it must be immediately placed into an endobag. The specimen should include, besides kidney and ureter, prostate and seminal vesicles in men and reproductive organs in women and lymph nodes.

Another important oncological aspect is the extent of lymphadenectomy. Extended lymph node dissection during radical cystectomy has previously been shown to be feasible for minimally invasive approach [17]. Barros et al. [18] were able to perform extended lymphadenectomy in patients who underwent simultaneous laparoscopic nephroureterectomy and cystectomy with a median of 14 lymph nodes. The mean number of lymph nodes we removed was 22.75. Laparoscopic approach presents equivalent oncological safety compared to open surgery $[19,20]$.

Open radical nephroureterectomy and radical cystectomy involve one large midline incision with higher morbidity and longer convalescence [21], but the laparoscopic approach implies very small trocar incisions and one incision about $4-5 \mathrm{~cm}$ to remove the specimen.

After repositioning the patient, in dorsal decubitus, we inserted the trocars for radical cystectomy using the camera trocar in the nephrectomy and the camera trocar became the working trocar. This technique of port placement allowed a safe laparoscopic procedure for radical cystectomy and pelvic lymphadenectomy in the second step of the surgery.

The specimen can be removed through a small lower midline incision [22], Pfannenstiel incision [18], or transvaginally in female patients [23]. We prefer to perform a small midline incision for the specimen removal because of the lower risk of evisceration.

There are small series of studies that conclude that performing in a single operation laparoscopic nephroureterectomy and cystectomy is feasible, with good oncological results and early recovery [24,25].

The main limitations of our study were the small number of cases (4 cases) and retrospective data collection. A large-scale prospective study will be necessary to provide more information on this surgery in the future.

\section{Conclusions}

In conclusion, the laparoscopic approach is widely spreading in uro-oncology and in some cases, it has become a standard of care. In selected cases, laparoscopic radical cystectomy and nephroureterectomy en bloc resection with lomboaortic and pelvic lymph node dissection are safe and feasible. It can be considered an alternative to open surgery, offering similar oncological and functional results and, in addition, better cosmetic results.

\section{References}

1. Siegel RL, Miller KD, Jemal A. Cancer statistics, 2016. CA Cancer J Clin. 2016;66:7-30.

2. Cosentino M, Palou J, Gaya JM, Breda A, Rodriguez-Faba O, Villavicencio-Mavrich H. Upper urinary tract urothelial cell carcinoma: location as a predictive factor for concomitant bladder carcinoma. World J Urol. 2013;31:141-145.

3. Li WM, Shen JT, Li CC, Ke HL, Wei YC, Wu WJ, et al. Oncologic outcomes following three different approaches to the distal ureter and bladder cuff in nephroureterectomy for primary upper urinary tract urothelial carcinoma. Eur Urol. 2010;57:963-969.

4. Stein JP, Lieskovsky G, Cote R, Groshen S, Feng AC, Boyd $\mathrm{S}$, et al. Radical cystectomy in the treatment of invasive bladder cancer: long-term results in 1,054 patients. J Clin Oncol. 2001;19:666-675.

5. World Health Organization (WHO) Consensus Conference on Bladder Cancer, Hautmann RE, Abol-Enein H, Hafez K, Haro I, Mansson W, et al. Urinary diversion. Urology. 2007;69(1 Suppl):17-49.

6. Margulis V, Shariat SF, Matin SF, Kamat AM, Zigeuner R, Kikuchi E, et al. Outcomes of Radical Nephroureterectomy: A Series From the Upper Tract Urothelial Carcinoma Collaboration. Cancer. 2009;115:1224-1233.

7. Witjes JA, Compérat E, Cowan NC, De Santis M, Gakis $\mathrm{G}$, Lebret T, et al. EAU Guidelines on Muscle-Invasive and Metastatic Bladder Cancer: Summary of the 2013 Guidelines. Eur Urol. 2014;65:778-792.

8. Clayman RV, Kavoussi LR, Figenshau RS, Chandhoke PS, Albala DM. Laparoscopic nephroureterectomy: initial clinical case report. J Laparoendosc Surg. 1991;1(6):343-349.

9. Parra RO, Andrus CH, Jones JP, Boullier JA. Laparoscopic cystectomy: initial report on a new treatment for the retained bladder. J Urol. 1992;148:1140-1144. 
10. Tang K, Li H, Xia D, Hu Z, Zhuang Q, Liu J, et al. Laparoscopic versus open radical cystectomy in bladder cancer: a systematic review and meta-analysis of comparative studies. PLoS One. 2014;9:e95667.

11. Khan MS, Challacombe B, Elhage O, Rimington P, Coker B, Murphy D, et al. A dual-centre, cohort comparison of open, laparoscopic and robotic-assisted radical cystectomy. Int J Clin Pract. 2012;66:656-662.

12. Veccia A, Antonelli A, Francavilla S, Simeone C, Guruli G, Zargar $\mathrm{H}$, et al. Robotic versus other nephroureterectomy techniques: a systematic review and meta-analysis of over 87,000 cases. World J Urol. 2020;38:845-852.

13. Rassweiler J, Godin K,Goezen AS, Kusche D, Chlosta P, Gaboardi F, et al. Radical cystectomy - pro laparoscopic. Urologe A. 2012;51: 671-678.

14. Liu F, Guo W, Zhou X, Ding Y, Ma Y, Hou Y, et al. Laparoscopic versus open nephroureterectomy for upper urinary tract urothelial carcinoma: a systematic review and meta-analysis. Medicine (Baltimore). 2018;97:e11954.

15. Berglund RK, Matin SF, Desai M, Kaouk J, Gill IS. Laparoscopic radical cystoprostatectomy with bilateral nephroureterectomy: initial report. BJU Int. 2006;97:37-41.

16. Fergany AF, Gill IS. Laparoscopic radical cystectomy. Urol Clin North Am. 2008;35:455-466.

17. Desai MM, Berger AK, Brandina RR, Zehnder P, Simmons M, Aron M, et al. Robotic and laparoscopic high extended pelvic lymph node dissection during radical cystectomy: technique and outcomes. Eur Urol. 2012;61:350-355.

18. Barros R, Frota R, Stein RJ, Turna B, Gill IS, Desai MM. Simultaneous laparoscopic nephroureterectomy and cystectomy: a preliminary report. Int Braz J Urol.
2008;34:413-421; discussion 421.

19. Ni S, Tao W, Chen Q, Liu L, Jiang H, Hu H, et al. Laparoscopic versus open nephroureterectomy for the treatment of upper urinary tract urothelial carcinoma: a systematic review and cumulative analysis of comparative studies. Eur Urol. 2012;61:1142-1153.

20. Navai N, Dinney CP. Oncologic Equivalence between Laparoscopic/Robotic and Open Radical Cystectomy. J Urol. 2016;195:1646-1647.

21. Hall MC, Womack S, Sagalowsky AI, Carmody T, Erickstad MD, Roehrborn CG. Prognostic factors, recurrence, and survival in transitional cell carcinoma of the upper urinary tract: a 30-year experience in 252 patients. Urology. 1998;52:594-601

22. Chłosta P, Drewa T, Obarzanowski M, Antoniewicz A, Borówka A. Do we reed a cosmetics effect for radical nephrectomy? Laparoendoscopic single-site surgery would help to answer this question. Videosurgery Miniinv. 2011;6:1-4.

23. Yuan LH, Chung HJ, Chen KK. Laparoscopic radical cystectomy combined with bilateral nephroureterectomy and specimen extraction through the vagina. J Chin Med Assoc. 2007;70:260-261.

24. Słojewski M, Chłosta P, Myślak M, Herlinger G, Dobroński $\mathrm{P}$, Kryst P, et al. Single-session laparoscopic cystectomy and nephroureterectomy. Wideochir Inne Tech Maloinwazyjne. 2013;8:158-161.

25. Tanaka Y, Okamura T, Chaya R, Nagai T, Kobayashi $\mathrm{D}$, Kobayashi $\mathrm{T}$, et al. Outcomes and Complications of Simultaneous Laparoscopic Cystectomy and Laparoscopic Nephroureterectomy with Umbilical Reduced Port Surgery. Asian Pac J Cancer Prev. 2018;19:3495-3500. 